\title{
Morphine injections in the taste aversion paradigm: Extent of aversions and readiness to consume sweetened morphine solutions
}

\author{
JUDITH E. GORMAN, RICARDO N. De OBALDIA, \\ ROBERT C. SCOTT, and LARRY D. REID \\ Bradley University, Peoria, Illinois 61606
}

\begin{abstract}
In three experiments, groups of rats were subjected to the procedures of classical taste aversion testing using morphine injections as the unconditioned stimulus. Subsequently, rats' consumption of sweetened morphine solutions was tabulated. In both tests of taste aversion and of readiness to consume sweetened morphine solutions, there were marked individual differences in rats' reactivity. The extent to which rats drank the flavored solution that had previously been paired with morphine injections was related to initial tendencies to consume sweetened morphine solutions. Extent of taste aversions established with lithium chloride, in a dose known to be poisonous, was greater than extent of aversions established with morphine. Extent of aversions following lithium injections was not related to consumption of morphine solutions. Rats' readiness to consume morphine solutions is probably related to initial negative consequences that may accompany assimilation of morphine.
\end{abstract}

In rats, following a particular taste, injections of a wide variety of agents producing unpleasantness or sickness will lead to aversion of the taste (Riley \& Baril, 1976). Interestingly, morphine can also be used to establish a taste aversion (Cappell \& LeBlanc, 1973; LeBlanc \& Cappell, 1974; Parker, Failor, \& Weidman, 1973). The fact that morphine can function as an unconditioned aversive stimulus potentially presents a conceptual difficulty for certain theorists. It poses a problem for those claiming that the rewarding or euphorigenic properties of addictive agents are critical to maintenance of behavior of assimilatoin of addictive agents.

In a study of morphine in the taste aversion paradigm, Farber, Gorman, and Reid (1976) noted considerable individual differences among rats in the extent of the aversion. Furthermore, across many pairings of the taste and morphine injections, a particular rat's responsiveness was relatively stable, i.e., a rat showing only slight aversion with first pairings also showed only slight aversion across a series of pairings, whereas a rat showing strong aversion with first pairings continued to show the strong aversion. It was suggested that the extent of the aversion might be related to readiness to consume

This study was supported by Grant DA01049 from the National Institute of Drug Abuse, DHEW. J. Gorman and L. Reid are now at the Department of Psychology, Rensselaer Polytechnic Institute, Troy, New York 12181. R. De Obaldia is now at the University of Oklahoma Health Sciences Center, School of Medicine, Department of Psychiatry and Behavioral Sciences, Oklahoma City, Oklahoma 73190. morphine. Consequently, in the present experiments, rats were first tested in the poison aversion paradigm and then given the opportunity to drink sweetened morphine solutions to see how responsiveness to morphine in the two circumstances might be related. Also, because informal data collections of our laboratory had indicated some differences between rats of the Holtzman and Sprague-Dawley strains, both strains of rats served as subjects.

\section{EXPERIMENT 1}

\section{Method}

Subjects and Apparatus. The subjects were 20, adult, male rats weighing from 275 to $300 \mathrm{~g}$ at the beginning of the procedure. Ten of the rats were purchased from Holtzman (Madison, Wisconsin) and 10 were from Sprague-Dawley (Madison, Wisconsin). They were housed in standard cages, in a room with constant lighting, and given access to commercially available food throughout the procedures and to water, except as specified. Water bottles equipped with ballpoint tubes were weighed to an accuracy of $0.5 \mathrm{~g}$ to determine the amount of fluid consumed.

Procedures. The rats were housed individually for at least 2 days before being deprived of water. Water was then presented for $15 \mathrm{~min} /$ day for 6 days. After this initial 6 days, the procedure was altered so that the next day and every 3rd day thereafter a $0.1 \%$ solution of saccharin was presented instead of water. Immediately after the presentation of saccharin solution, all subjects were injected intraperitoneally with $10 \mathrm{mg} / \mathrm{kg}$ morphine sulfate $(10 \mathrm{mg}$ of morphine as a salt in $1 \mathrm{ml}$ of physiological saline). The regimen continued through four presentations of the saccharin solution; consequently, there was one measure of rats' reactivity to saccharin prior to injections and there were three measures subsequent to morphine injections.

On the day after the last test in the poison aversion paradigm, the rats were presented with both water and a sweetened morphine solution for 4 days. The sweetened morphine solution was $20 \%$ 
sucrose $(\mathrm{w} / \mathrm{v})$ with $0.5 \mathrm{mg}$ of morphine sulfate for every milliliter of solution. Then rats were given only the sucrose-morphine solution for 4 days, followed by another 4 days of choice between water and sucrose-morphine solution. Subsequent to the second 4 days of choice, both water and a sucrose-morphine solution were presented daily, but the concentration of the sucrose was reduced each day by $2 \%$. Thus, on the 10th day of this regimen with decreasing sucrose, the rats were presented with water and with water with $0.5 \mathrm{mg} / \mathrm{ml}$ of morphine. Following the above procedures, the rats were given only food and water for 8 days, $24 \mathrm{~h} / \mathrm{day}$, and then presented with food, water, and the $20 \%$ sucrose solution with $0.5 \mathrm{mg} / \mathrm{ml}$ of morphine for 4 days. This last presentation of morphine was a test of relapse.

\section{Results and Discussion}

Figure 1 summarizes the poison aversion test. As shown, the Holtzman rats, on the average, consumed more water prior to injections and more saccharin both before and after initiation of morphine injections. The $t$ test comparing mean water consumption of the strains yielded a $\mathrm{t}(18)=2.26$, $\mathrm{p}<.05$. An ANOVA on repeated measures with one factor being strain of rats and another the scores of each day's presentation of saccharin yielded for Strain an $F(1,18)=7.8, p<.05$. As shown by Figure 1 and the ANOVA, the rats decreased their consumption of the saccharin solution with successive presentations, $F(3,54)=17.0, p<.0001$, indicating a substantial poison aversion. There was not a reliable interaction between Strain and Days, $F(3,54)$ $=1.3, \mathrm{p}<.25$, indicating no reliable differential reactivity with the advent of morphine injections. As with the subjects of Farber et al. (1976), there were considerable individual differences in extent of the aversion, e.g., rats on fourth presentation of saccharin drank from 1 to $17 \mathrm{~g}$ of the test solution. The rats that showed the strongest aversions after the first injections also showed the strongest aversions after the last injections, Kendall's coefficient of concordance, $\mathrm{W}$, for the three tests following morphine injections $=.63, \chi^{2}(19)=35.9, \mathrm{p}<.02$.

Figures 2 and 3 depict data on consumption of the sweetened morphine solutions for each strain. Figure 2 presents mean daily consumption of the morphine solution with the first 4 days of choice between tap water and the solution. The leftmost data points of Figure 3 are summaries of the data of Figure 2.

As can be deduced from Figure 2, the rats did take, on the average, considerable quantities of the morphine solution during its initial presentation. An ANOVA on repeated measures of the data of Figure 2 yielded for the factor of Strain an $F(1,18)=$ $5.6, \mathrm{p}<.05$, for the factor of Days an $F(3,54)=$ $4.8, \mathrm{p}<.005$, and for the interaction an $F(3,54)$ $=1.6, \mathrm{p}<.25$.

The rats of the Holtzman strain consumed more fluids in general than the rats of the Sprague-Dawley strain. This difference may be due to their different b@dy weights. The Holtzman rats weighed a mean

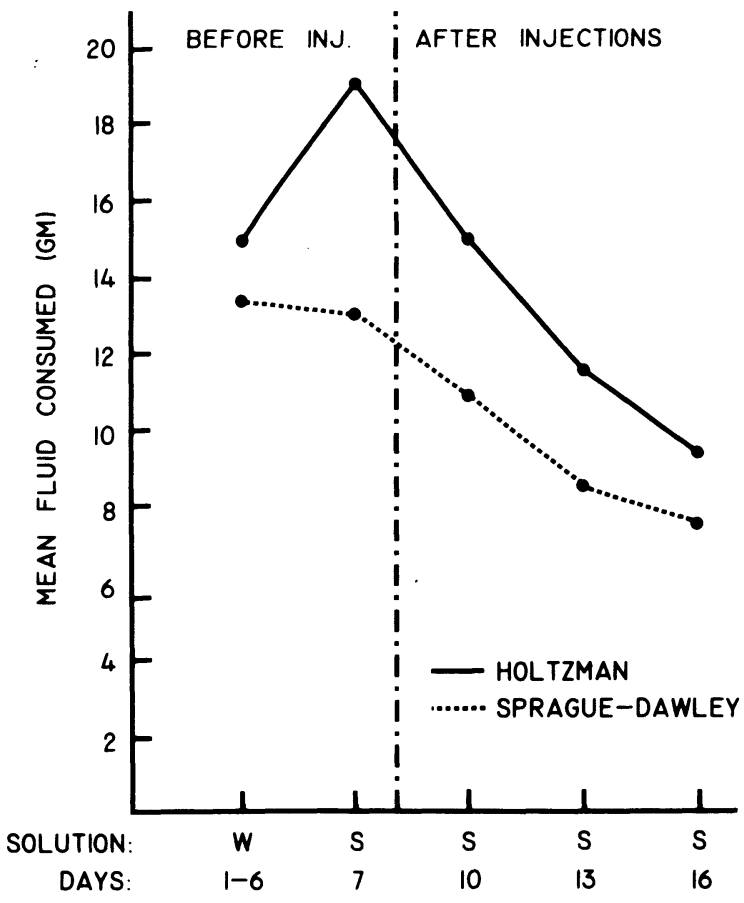

Figure 1. Mean grams (roughly milliliters) of fluid consumed during 15-min daily sessions. $W=$ tap water as the fluid; $S=$ saccharin solution as the fluid. Days refers to consecutive days of the procedure. The data-point for " $W$ " is the mean of 6 days of measurement. Injections of morphine followed the first presentation of saccharin solution as well as subsequent presentations. Consequently, the 2 nd day with saccharin solution is the first potential index of morphine's effects.

of $362 \mathrm{~g}$ on the 1st day of saccharin presentation (taste aversion paradigm), while the Sprague-Dawleys weighed $339 \mathrm{~g}, \mathrm{t}(18)=5.4, \mathrm{p}<.001$. Although there were reliable differences between the strains in initial consumption of sweetened morphine solutions, that difference may not reflect differential reactivity to morphine. The rats of the Holtzman strain were consuming more fluids and weighed more than the rats of the Sprague-Dawley strain, and these factors may account for the differences in consumption.

Across all rats, there were considerable individual differences in amounts of sweetened morphine solution consumed, e.g., rats drank from 4 to $59 \mathrm{~g}$ during the 1 st day of presentation and from 2 to $80 \mathrm{~g}$ during the 4th day. Rats seemed to maintain their relative standing across the 4 days of initial choice, Kendall's W $=.81, \chi^{2}(19)=61.6, p<.001$.

Figure 3 presents mean consumption of morphine solution across the conditions in which it was presented. Analyses of the data for conditions after the initial choice failed to provide any evidence for concluding that differences between strains were reliable. Consequently, some of the further analyses ignore the designation of strains.

Subsequent to the first 4 days of choice, the rats' 


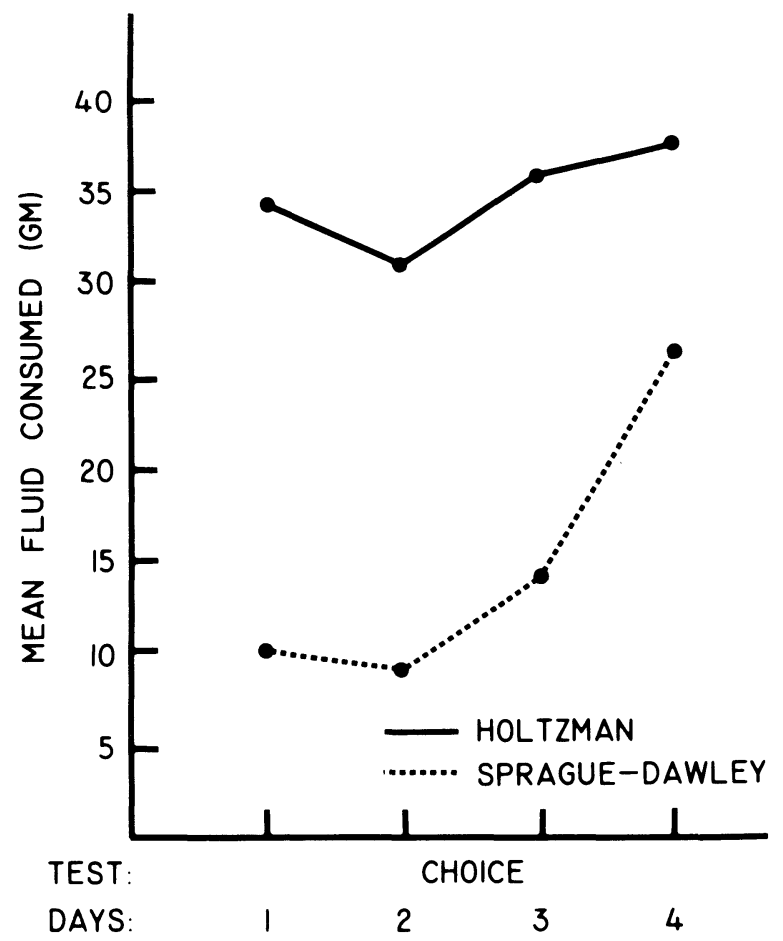

Figure 2. Mean grams $(1.08 X=\mathrm{ml})$ of sweetened morphine solution taken daily by the two strains of rats. The rats always had water available, so the condition was labeled "choice" because they could meet fluid needs by taking water. Days refer to consecutive days of the procedures associated with oral consumptions of morphine solutions.

only source of fluids was the $20 \%$ sucrose solution spiked with morphine (the condition labeled "forced" in Figure 3). This condition increased consumption by all rats; comparing mean amount taken during first choice to mean amount taken during the "forced" condition yields a $\mathrm{t}(18)=4.7, \mathrm{p}<.001$. On the average, the rats' mean consumption during the "forced" condition was greater than that needed to prevent dehydration, indicating that the rats were taking the solution for its morphine. This idea is confirmed by the data of the second choice procedures, during which rats maintained high levels of consumption when they had water available. A t test comparing mean consumption during initial choice period to that during second choice period yielded $t(18)=5.9, p<.001$. The correlation between mean consumption of the two choice conditions $=.44$, $\mathrm{p}<.05$. Furthermore, during the days when sucrose concentrations were reduced, the rats maintained high levels of consumption until the concentration of sucrose was at about $4 \%$.

All rats, with the exception of two rats of the Sprague-Dawley strain, stopped taking quantities of the morphine solution when the sucrose concentration was $2 \%$ or $0 \%$. The two that continued to take the morphine solution drank 41 and $46 \mathrm{~g}$ of the solution at $0 \%$ sucrose, or 101 and $68 \mathrm{mg} / \mathrm{kg} /$ day of morphine. Usually, rats abruptly stopped taking the solution with a given reduction in sucrose rather than gradually reducing intake as sucrose concentration was reduced. When the rats stopped taking quantities of morphine solution, they showed rapid weight loss. They lost as much as $69 \mathrm{~g}$ of body weight across a 2-day period and a mean of $25 \mathrm{~g}$ across 2 days (from data of rats taking greater than $20 \mathrm{~g}$ of solution/day toward the end of choice with decreasing sucrose).

After 8 days of no opportunity to take morphine (after the $0 \%$ sucrose conditions), the rats were again given an opportunity to take a $20 \%$ sucrosemorphine solution, the condition labeled "relapse" in Figure 3. The tendency to relapse to taking

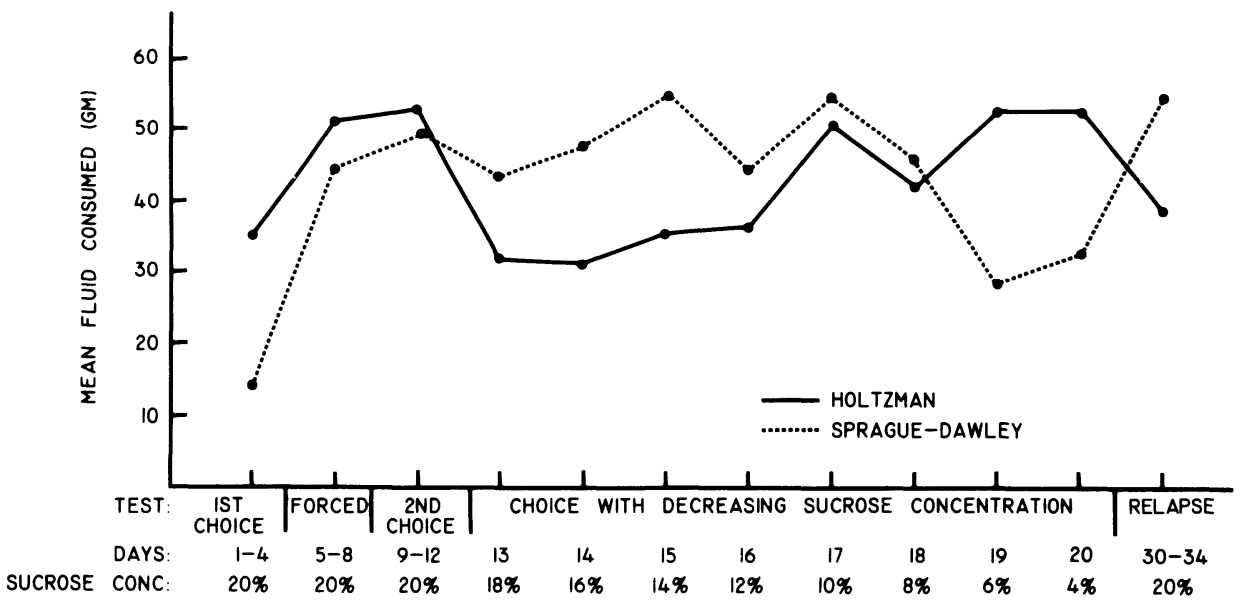

Figure 3. Mean grams of sweetened morphine solution taken by the two strains of rats across the days and procedures of these measures. Choice refers to the circumstances in which rats had water as well as the morphine solution available. Forced refers to the circumstances in which rats had only the morphine solution available. Relapse refers to the circumstance of choice after days of abstinence from morphine. 
morphine after abstinence is of particular interest because it is the measure that is analogous (if any measure is analogous) to a salient measure of treatment outcome in programs treating human addicts. The rats of this procedure took means of $28,56,50$, and $55 \mathrm{~g}$ of sweetened morphine solution during each of the respective days of relapse, Kendall's $\mathrm{W}=.79, \chi^{2}(19)=53.7, \mathrm{p}<.001$. A comparison of mean consumption across the initial 4 days of opportunity to consume morphine solution (first choice) to mean consumption of last 4 days of opportunity (relapse) yielded a $\mathrm{t}(18)=3.1, \mathrm{p}<.005$, indicating, of course, that a history of assimilation of morphine potentiates taking morphine. The correlation between the scores of the first choice period and relapse, however, was only .04, indicating that initial level of consumption and consumption after a history of morphine assimilation are probably unrelated. The correlation between the mean relapse score and the mean scores of the forced period was $.38, p \approx .05$, and between the mean relapse score and the mean of the second choice period was $.30, p>.05$. The highest correlation between relapse scores and any other measure of consumption of Figure 3 was the correlation of $.52, \mathrm{p}<.05$, between relapse and consumption with the $8 \%$ sucrose-morphine solution.

Our hypothesis is that some index of poison aversion will predict rats' consumption of sweetened morphine solutions. Since there were high levels of concordance among consumption scores for a given procedure (e.g., first choice and relapse), the mean across the days of that procedure was taken as representative of rats' performances. There are a number of potential indices of extent of poison aversion. The following four indices of aversion are intuitively reasonable: (1) grams of saccharin solution taken on last (4th) day of presentation, (2) the mean grams of saccharin solution taken across the last three presentations, (3) the difference in grams consumed on first presentation of saccharin solution compared to grams consumed on last presentation, i.e., the difference between saccharin solution taken before and after morphine injections, and (4) the difference in grams consumed on first presentation of saccharin compared to mean of grams across the last days of presentation.

Using each of these potential indices of extent of poison aversions, we attempted to predict initial consumption of sweetened morphine (mean of first choice period) with data of each strain of rats. In general, using any one of the indices led to the same conclusion, but the simple measures of last day of consumption of saccharin did a somewhat better job. Consequently, mean grams consumed on last day of saccharin presentation (fourth presentation) was used as the index of extent of poison aversion.
For the rats of the Holtzman strain, the correlation between extent of poison aversion and initial consumption of morphine solution was $r=.83, p<.005$, i.e., the more the consumption during poison aversion testing, the more the consumption of the morphine solution. For the rats of the SpragueDawley strain, none of the indices of poison aversion was correlated reliably to their initial consumption of the morphine solution. The lack of a relationship among the scores of the Sprague-Dawley rats was due primarily to two subjects whose pattern of responding did not conform to the pattern of responding of the Holtzman rats.

The next question addressed was whether the selected index of poison aversion was the most salient predictor of initial consumption of morphine solution or whether the poison aversion index was correlated to some more meaningful variable. Furthermore, is it possible that the index in combination with some other variable more adequately predicts initial consumption? Other potentially relevant variables are mean water consumption prior to presentation of saccharin, mean water consumption on days between saccharin consumption, and rats' body weights. Initial consumption was regressed on the poison index paired with each of these variables for data of each strain. For the scores of the Holtzman rats, the index of aversion was reliably related to initial consumption in each regression using the other variables, all $F(1,7) s>12.6$, ps $<.01$. None of the other potential independent variables was reliably related to initial consumption of sweetened morphine. Furthermore, no variable in combination with the index of poison aversion added appreciably to predictability of initial consumption. Similar analyses with the scores of the SpragueDawley rats yielded findings to suggest that their data would not predict initial consumption of sweetened morphine solutions.

The extent of poison aversion did not predict consumption during days and procedures following the initial choice period. The correlation between extent of poison aversion (last day of saccharin consumption) and mean relapse scores, for example, was only -.14 .

The results (Figure 3) show that it is possible, once rats have taken quantities of the sweetened morphine solution, to gradually reduce the sucrose concentration to about $6 \%$ and still have large quantities of the solution consumed. Some rats even maintain their consumption of $0 \%$ sucrose consuming morphine even though the taste is, evidently, extraordinarily aversive to rats. These observations, plus others of our laboratory, lead to the suggestion that it is possible to gradually fade out the sucrose and still have high levels of consumption. It is possible to phase out the sucrose relatively rapidly if a $6 \%$ 
sucrose concentration is the desired goal, but the reductions in sucrose will probably have to be very gradual subsequently to get a large portion of the rats to continue consumption with no sucrose. All of our observations suggest that the $6 \%$ sucrosemorphine solution is too bitter to be taken by rats unless they are inured to it.

The model of oral consumption of sweetened morphine solutions has the advantage of ease of implementation, thereby allowing large numbers of rats to be tested in moderately financed laboratories. The model with the feature of reducing sucrose concentrations has interesting features. The rats choose to consume in spite of the bitterness of morphine, demonstrating high motivation. The rats show severe withdrawal symptoms when consumption is suspended, and they show a marked tendency to relapse after considerable time away from the opportunity to consume. The rats demonstrate individual differences that are interesting and meaningfully related to other potentially relevant variables.

\section{EXPERIMENT 2}

In Experiment 1, both the solutions used (the saccharin of aversion testing and the sweetened morphine solution) were potentially sweet solutions. Consequently, the rats may have been merely reacting, in both instances, to sweetness and not to morphine. In this experiment, the solution used as the conditional stimulus in the taste aversion paradigm was flavored with almond extract.

\section{Method}

Eleven Holtzman and 11 Sprague-Dawley rats were used in Experiment 2. They were tested for an aversion to a novel solution and initial consumption of a sweetened morphine solution (daily consumption across 4 days of $20 \%$ sucrose with $0.5 \mathrm{mg} / \mathrm{ml}$ of morphine sulfate). The procedures of the two tests were nearly identical to the tests of Experiment 1 . However, instead of the $0.1 \%$ saccharin solution used as the conditional stimulus in Experiment 1 with poison aversion testing, the conditional stimulus in Experiment 2 was an almond-flavored solution. The almond flavoring was a commercially available almond extract (water, $35 \%$ alcohol, and oil of almond) used for flavoring foods, and $4 \mathrm{ml}$ of flavoring was mixed with $3000 \mathrm{ml}$ of tap water.

\section{Results and Discussion}

Results of the two tests, one of poison aversion and one of the readiness to consume sweetened morphine solutions, are summarized in Figures 4 and 5 . An ANOVA on the data of each day's presentation of almond solution did not support the notion that a poison aversion was established, i.e., consumption was not reliably reduced with injections. Rats' consumptions of a novel solution, however, typically increase in this paradigm if saline injections are given (Farber et al., 1976). Despite the fact that on the average there were no indications of reduced drinking of the almond solution, there were marked

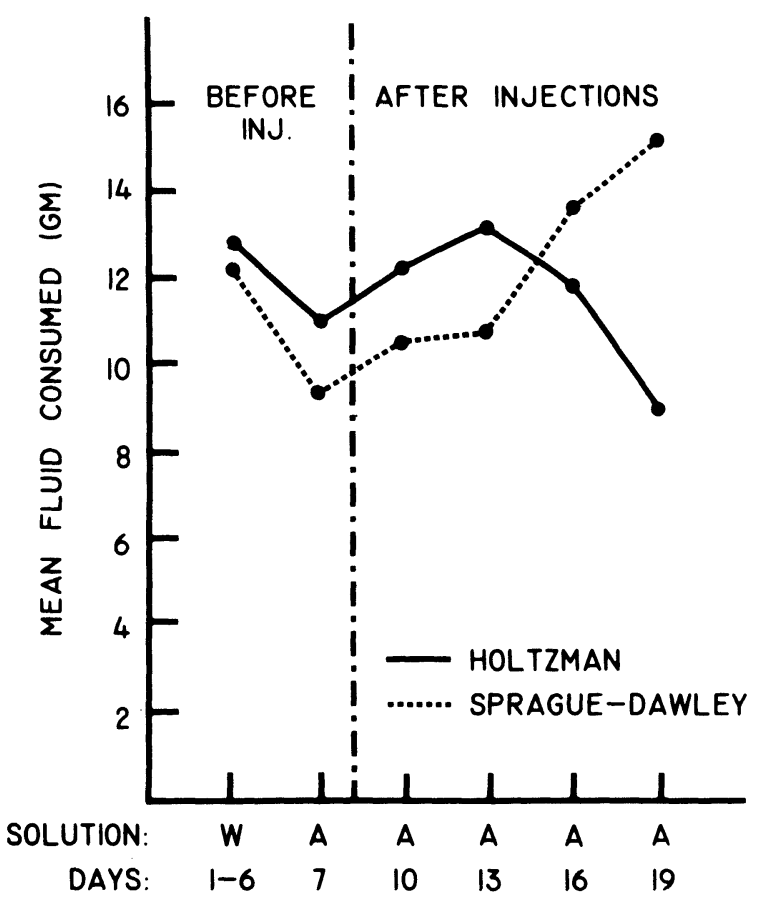

Figure 4. Mean grams of fluid consumed during 15-min daily sessions. $W=$ tap water as the fluid; $A=$ almond flavored solution as the fluid. Days refers to consecutive days of the procedure.

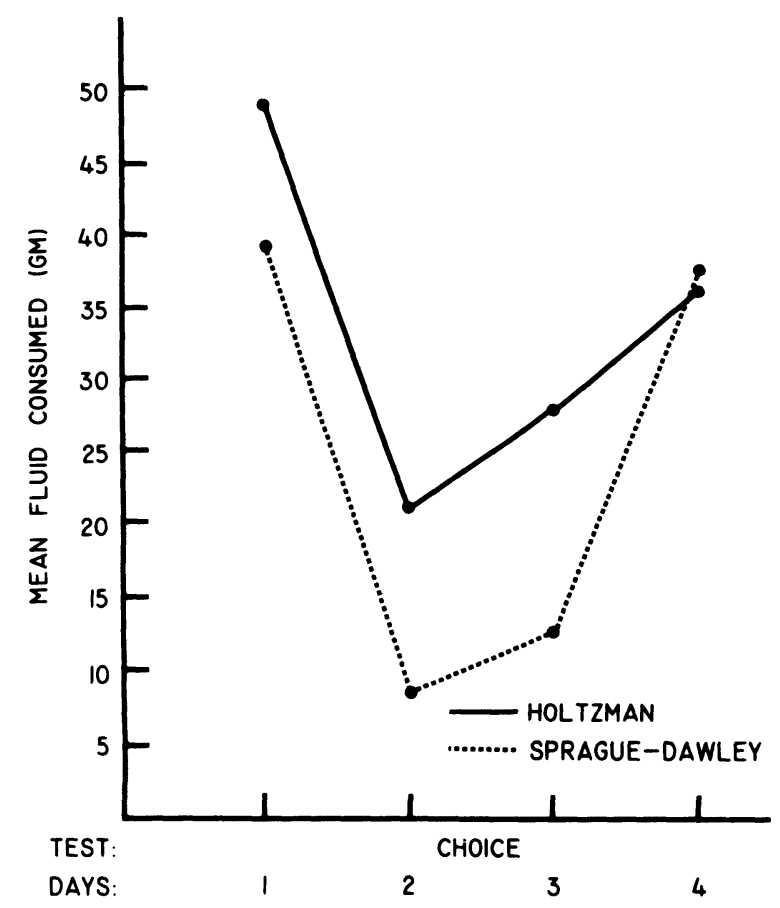

Figure 5. Mean grams of sweetened morphine solution taken daily by the two strains of rats. 
individual differences among the rats. For example, two Holtzman rats' consumption was less than $8 \mathrm{~g}$ on the fourth presentation whereas two of the same strain took more than $21 \mathrm{~g}$.

The ANOVA for the data germane to Figure 5 (consumption of sweetened morphine) yielded for the factor of Strain an $F(1,20)=1.92, p<.25$; for the factor of Days an $F(3,60)=25.0$, p $<.001$; and for the interaction an $F(3,60)=1.82, p<.25$. The rats of the Holtzman strain were, on the average, more avid consumers of the sweetened morphine solution, although not reliably greater consumers. Their higher consumption cannot be attributed this time, however, to their heavier weight since the Holtzman rats were lighter than the Sprague-Dawley rats, $\mathrm{t}(18)=1.87, \mathrm{p}<.05$.

Using the index of poison aversion derived in Experiment 1 (grams consumed 4th day of presentation of conditional stimulus), the extent of poison aversion was correlated to mean daily consumption of sweetened morphine, $\mathrm{r}=.73, \mathrm{p}<.005$, for the scores of the Holtzman rats. The same type of multiple regression analyses as performed with the data of Experiment 1 confirmed that the index of poison aversion was the salient variable in predicting consumption of the morphine solution for the Holtzman rats and that potential combinations of variables added little to the ability to predict. As with the results of the Sprague-Dawley rats of Experiment 1 , there were no reliable relationships between measures of poison aversion and consumption of the morphine solution for the data of Sprague-Dawley rats of this experiment.

The lack of a strong poison aversion with almond flavoring and morphine injections is probably due to poor discrimination, on the part of some rats, between the almond solution and the tap water. Nevertheless, some feature of testing for poison aversions was evidently salient for the Holtzman rats and the measures did predict subsequent consumption of sweetened morphine solution. It is difficult to maintain in light of results of this experiment that the relationships between reactivity in the taste aversion paradigm and in the consumption paradigm, when observed, are due to the similarity of tastes in the two tests. This conclusion is strengthened by observations in Experiment 3.

\section{EXPERIMENT 3}

It is clear that morphine can be used to establish an aversion in the taste aversion paradigm. The extent of those aversions, however, is difficult to assess because there are no direct comparisons of aversions established with morphine and those established with a standard poison. An injection of lithium chloride $(\mathrm{LiCl})$ is commonly used as the unconditioned aversive stimulus in the taste aversion paradigm. Behavior following $\mathrm{LiCl}$ injections, therefore, can serve as a standard to compare behavior following morphine injections. From a pilot study (Bush, 1976), there was some indication that inserting an electrode into the hypothalamus modified the extent of poison aversions using morphine. Consequently, in this experiment we also tested the effects of electrode insertion.

\section{Method}

Subjects. Forty-seven (five groups of eight rats each and one group of seven rats), experimentally naive, adult, male SpragueDawley rats served. They were housed individually and given access to food throughout the procedures and water according to specified schedules.

Procedure. Three days after arrival at the laboratory from the commercial supplier, at which time they weighed a mean of $223 \mathrm{~g}$, all rats received the procedures of surgery. Using standard surgical procedures, one-third of the rats had an electrode inserted unilaterally into the lateral hypothalamus. The bipolar electrode was made by Plastic Products and was Teflon-coated (each wire was $.25 \mathrm{~mm}$ in diameter). With bregma and lambda on the same horizontal plane, the coordinates of the lateral hypothalamic insertions were $3.3 \mathrm{~mm}$ posterior to bregma, $1.5 \mathrm{~mm}$ lateral to the midline, and $8.5 \mathrm{~mm}$ from the top of the skull. Another third had electrodes inserted unilaterally into the caudate nucleus, at bregma, $2.5 \mathrm{~mm}$ lateral, and $5.8 \mathrm{~mm}$ deep. Just after an electrode insertion, the electrode was removed, the hole filled with bone wax, and the wound sutured. The other third had all the procedures except drilling the hole for the electrode and, of course, the insertion of an electrode.

For 2 days subsequent to surgical procedures, all rats always had access to water. For 6 days, thereafter, they were given water for only $15 \mathrm{~min} /$ day. During this time, the rats learned to take a daily ration of water, and they maintained or gained weight during the last of the 6 days. Rats then continued to get their fluid during 15-min periods a day for 18 days, as before, except that every 3rd day the fluid was a $.1 \%$ saccharin solution instead of the usual tap water. Immediately following each presentation of saccharin solution, one-half of the rats received an intraperitoneal injection of $12 \mathrm{ml} / \mathrm{kg}$ of $\mathrm{LiCl}$ solution $(.15 \mathrm{M}$ solution or $6.359 \mathrm{~g} /$ liter). The other half received a subcutaneous injection of morphine, $20 \mathrm{mg} / \mathrm{kg}$ (the solution was mixed so that injections were $1 \mathrm{ml} / \mathrm{kg}$ ). Consequently, there was one measure of consumption of saccharin prior to potential conditioning and five measures afterwards. Four days subsequent to the testing in the poison aversion paradigm, the rats were given an opportunity to consume, throughout the day, either an $18 \%$ sucrose solution spiked with $0.5 \mathrm{mg} / \mathrm{ml}$ of morphine sulfate or tap water for 5 days.

\section{Results and Discussion}

Figure 6 summarizes the results of poison aversion testing. An ANOVA (repeated measures across the measurements of 6 days of presentation of saccharin) yielded an $F(1,41)=27.2, p<.001$, for the Type of injection (morphine vs. lithium); an $F(5,205)=$ $63.5, p<.00001$ for the factor of Days, and an $F(5,205)=9.9, p<.001$, for the interaction of Type of drug by Days. No other factors approached standards for concluding they represented reliable sources of variance. Student $t$ tests for correlated differences using data of 1st day of saccharin 


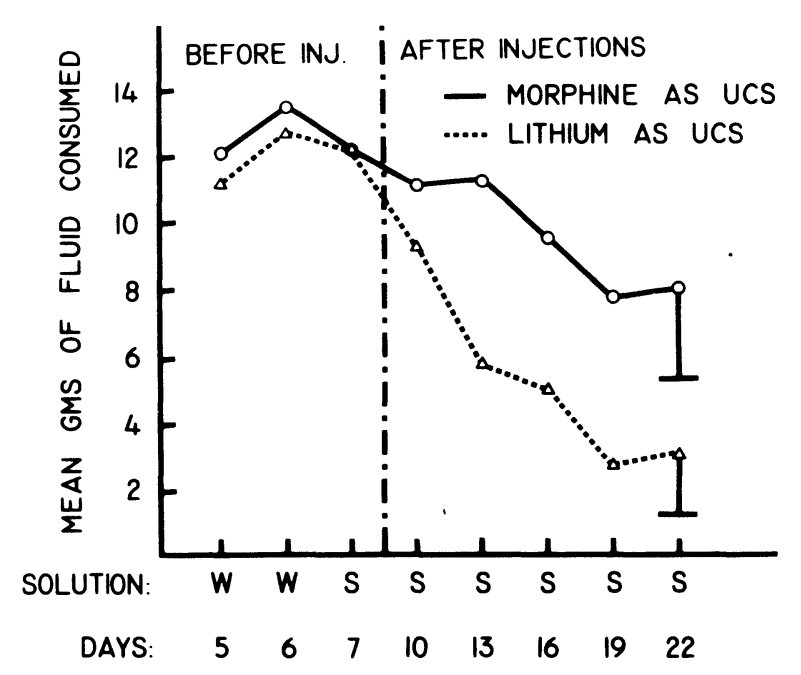

Figure 6. Mean grams of fluid consumed during 15-min daily sessins. $W=$ tap water as the fluid; $S=$ a saccharin solution as the fluid. One group had morphine injections as the unconditioned stimulus in the poison aversion paradigm, while another group had lithium as the UCS. The length of line from the data point reflects size of the standard deviation of the scores. Days refers to days of the procedure.

presentation (day prior to first injection) and data of last day of saccharin presentation (after five pairings of saccharin and effects of injections) yielded $t(22)=4.0, p<.001$, for rats of morphine injections and $\mathrm{t}(23)=12.1, \mathrm{p}<.001$, for rats of lithium injections. These results confirm that aversions were established by the injections as indexed by reduced consumption. The $t$ test comparing scores of rats of morphine vs. those of lithium on the 1 st day of saccharin presentation was $\mathrm{t}(45)=0.6, \mathrm{p}>.25$; on the last day of saccharin presentation $t(45)=6.6$, $p<.001$. Since the groups did not differ prior to injections (1st day of saccharin presentation), it is presumed that the differences seen on the last day of saccharin presentation were an effect of the type of injection. The reliable interaction factor and the other analyses lead to the conclusion that the lithium injections led to reliably less consumption or considerably greater aversion.

Represented in Figure 6 are the standard deviations for scores of last presentations of saccharin solution. The variability of the scores of the group with morphine injections is reliably larger than those with the lithium injections $[\mathrm{F}(22,23)=2.81, \mathrm{p}<.01]$. Some rats with morphine injections hardly reduced their intake of the test-solution, e.g., S-565 drank $12.0 \mathrm{~g}$ on a day of water presentation, drank $13 \mathrm{~g}$ on the 1st day of saccharin presentation, and $12.5 \mathrm{~g}$ on the 6th day of saccharin presentation. Other rats with morphine injections showed as much reduction in consumption as rats with lithium injections.

Figure 7 depicts results of tests with sweetened morphine solution for the two groups that previously had received either morphine or lithium injections during poison aversion tests. An ANOVA of daily scores (grams) of consumption with the subjects grouped as with the poison aversion analyses was done. This repeated measures ANOVA had factors associated with whether the rats had morphine or lithium injections, with the three surgical procedures, and with the five daily measures of amount of sweetened morphine solution consumed. The factor of Type of injection (morphine-lithium) had an $\mathrm{F}(1,41)=27.2, \mathrm{p}<.001$; the factor of Days of repeated measurement had an $F(5,205)=9.9$, $\mathrm{p}<.001$. No other factors approached standards for statistical significance. Student $t$ tests were used for further summaries of the data. The $t(45)$ between the scores of rats of morphine and of rats of lithium for each of the 5 days of consumption were .67, $.78,2.38,2.03$, and 1.82 , respectively. The $p$ values for differences of Days 3 and 4 are $<.05$; the value for Day 5 is $<.10$. The lack of a reliable difference on the first 2 days of consumption is of interest with respect to interpretation of the data. The $t$, for correlated measures, comparing Day 1 to Day 5 consumption for the two groups yielded $t(22)$ for the rats of morphine $=1.0, p<.12$, and $t(23)$ for rats of lithium $=3.5, \mathrm{p}<.01$, i.e., rats that previously had had morphine injections did not reliably change their consumption from the 1st to the last day of testing, whereas the rats of lithium injections reliably reduced their consumption on Day 5 compared to Day 1 of consumption of sweetened morphine solutions.

None of the analyses support a suggestion that the procedures of surgical intervention affected the rats'

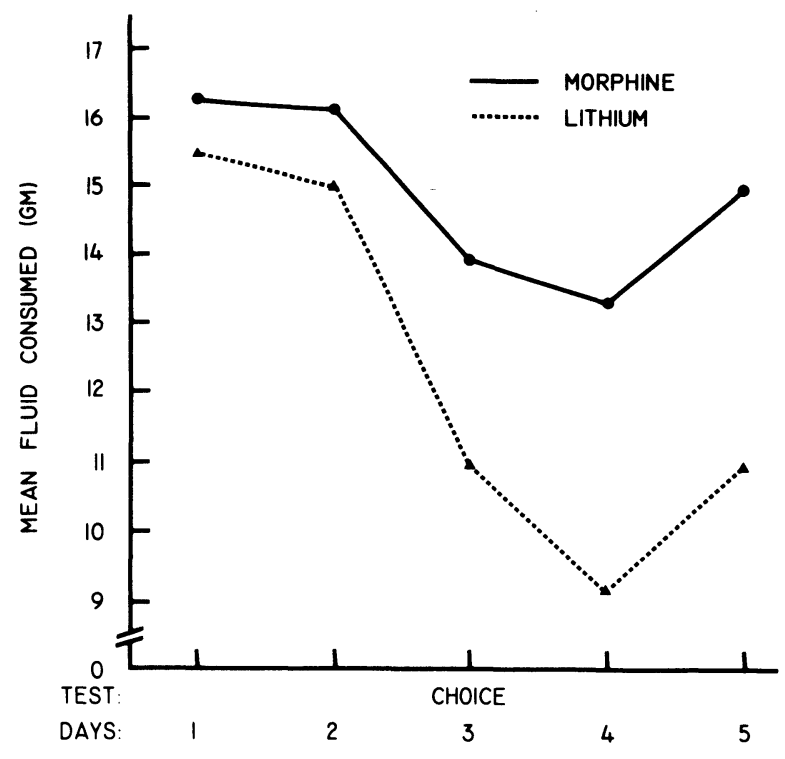

Figure 7. Mean consumption of sweetened morphine solution by the two groups of rats. One group previously had morphine injections and one group previously had lithium injections. 
performances. Consequently, all further analyses ignore the distinction of surgical procedures. Although data of a pilot study has indicated that the surgical intervention might affect the outcomes of our tests, this more formal test leads to the conclusion that the surgical procedures have negligible effects. This finding lays the foundations for tests involving intracranial stimulation and larger central nervous system lesions with the measures used in these procedures.

The analyses of Experiments 1 and 2 suggested that the fourth measure with the conditional stimulus was tine score that best predicted subsequent consumption of sweetened morphine. Consequently, a correlation was calculated between the fourth measurement with saccharin of rats getting morphine injections (a poison aversion index) and the mean of the first 4 days of consumption of sweetened morphine; this was $.73, p<.005$. A similar correlation for rats given lithium was .22 ( $p>.05)$.

In this experiment, the Sprague-Dawley rats' extent of poison aversion was clearly related to their initial consumption of sweetened morphine solutions, whereas the extent of poison aversion and consumption of morphine solution of SpragueDawleys was not in Experiments 1 and 2. The dose of morphine in the poison aversion paradigm of this experiment was larger, and this difference probably accounts for the differences in results across experiments.

The fact that the 1st day's consumption of sweetened morphine solution was relatively large and was roughly equal for the groups previously receiving morphine and lithium forms the basis of a conclusion that the taste of saccharin and sweetened morphine solutions were not similar. If they were similar, it would be expected that the aversion would have generalized from the tests with saccharin to the tests with sweetened morphine. Since the rats of $\mathrm{LiCl}$ injections showed intense aversions to the saccharin solution, it would be expected, if they were reacting to sweetness, that they would have avoided the sweetened morphine solution on the 1 st day of its presentation. The subsequent slight, but probably reliable, reduction in consumption of the rats of lithium injections and the trend for rats of morphine injections to not decrease their consumption leads to the suggestion that the morphine injections of the poison aversion procedures potentiated subequent consumption of sweetened morphine.

\section{DISCUSSION}

Using the procedures specified by Edwards (1976), a test was done for the homogeneity of the five correlations (two derived from Experiment 1, two from Experiment 2, and one from Experiment 3) specify- ing the relationship between index of poison aversion using morphine as the unconditioned stimulus and initial consumption of sweetened morphine. That test yielded a $\chi^{2}(4)=15.3, p<.01$, indicating that the various correlations cannot be considered estimates based on samples drawn from the same population. Nevertheless, the overall correlation between the poison aversion index and initial consumption is about .55 (calculated across all scores or taking a weighted average of the values associated with the separate rs). It is obvious that the two correlations from the Sprague-Dawley rats of Experiments 1 and 2 are discrepant from the other three values, since the two values are not reliably different from zero and the three values are $.83, .73$, and .73 , specifying strong, significant relationships.

The procedues for the Sprague-Dawley rats of Experiments 1 and 2 compared to the procedures of the Sprague-Dawley rats of Experiment 3 are very similar, except that the rats of Experiment 3 were given larger doses of morphine in the poison aversion paradigm. The Holtzman rats, on the average, take more morphine solution during its initial presentation than Sprague-Dawley rats and their responsiveness in the poison aversion paradigm predicts their initial consumption using a $10 \mathrm{mg} / \mathrm{kg}$ dose of morphine, whereas responsiveness in the poison aversion paradigm with the same dose does not predict initial consumption with Sprague-Dawley rats. These differences are related to strain, but may or may not be related to the strains' reactivity to morphine. The Holtzman rats take more fluids and may be less sensitive to tastes, and these experiments confound these potentially relevant variables with strains' reactivity to morphine.

The relationship between reactivity in the poison aversion paradigm and initial consumption of sweetened morphine is not, however, a product of generalization of similar tastes of the fluids used in the two procedures. The same relationship was observed when two different solutions were used as the conditional stimulus in the poison aversion paradigm. Furthermore, the first day of consumption of sweetened morphine was not a function of reactivity to the saccharin solution when lithium was used as the unconditioned stimulus, nor was the first day of consumption of sweetened morphine solution reliably different between the two groups being conditioned to avoid saccharin, one with morphine and one with lithium injections. Nor was the reactivity in the two tests of a strain related to amount of water consumption or body weight. Indeed, the extent of consumption of a fluid used as a conditional stimulus on the fourth presentation (or fifth or sixth presentation) following a heavy dose of morphine in the poison aversion paradigm seems to be related to initial consumption of sweetened morphine solutions, although the relationship may not be 
seen in groups of Sprague-Dawley rats unless the dose of morphine, used as an unconditioned stimulus, is large.

It may not surprise anyone to learn that a substance that has a tendency to make an individual ill (produce a poison aversion) will also not be taken avidly when mixed with sweet water. There are, nevertheless, some important implications that can be derived from this rather simple demonstration. The finding that morphine can be used to establish a poison aversion can be used as evidence that the behavior of morphine assimilation is not maintained by the positively reinforcing attributes of morphine assimilation. In light of the data of these experiments, however, the results showing that morphine can be an aversive stimulus do not provide inordinate conceptual difficulties for theories stressing the positively reinforcing characteristics of addictive agents as the critical features maintaining the behavior of assimilation of the agents.

The reasons that the findings of poison aversion tests are not contrary to theories that stress the positive reinforcing properties of morphine are stated here. First, the extent of aversions is less than that of a standard poison, with some subjects showing little or no aversions (Experiment 3). Second, subjects showing minimal aversions are typically the most avid consumers (Experiments 1, 2, \& 3). Third, the aversive consequences that might immediately follow morphine intake wane with extensive assimilation of morphine (LeBlanc \& Cappell, 1974). The aversive consequences can evidently wane very rapidly with daily consumption, since rats escalate their intake across days rather rapidly, but wane rather slowly with single injections a day (Farber et al., 1976). Fourth, the aversive consequences of an injection probably emerge during a relatively short period after an injection, but subsequently there is a prolonged period during which positive affect predominantes (Farber \& Reid, 1976; Rossi $\&$ Reid, 1976). Mixed affective events as contingencies, such as brief negativity followed by positivity, are capable of maintaining relatively high rates of behavior (Casper \& Reid, 1975). Fifth, the negativity is probably greater with imposed injections than in circumstances where the dose is regulated by the individual.

There is no doubt that morphine can be used to establish a poison aversion. It is doubtful, however, whether this fact is relevant to the question of what maintains the behavior of morphine assimilation. The relationship between morphine's capacity to establish a poison aversion and intake of morphine is limited to initial intake. Furthermore, the greater the aversion, the less the initial intake, but after assimilation of quantities of morphine even that relationship breaks down.

\section{REFERENCES}

Bush, M. A. Unilateral brainstem lesions and morphine consumption. Unpublished MA thesis, Bradley University, 1976.

Cappell, H., \& LeBlanc, A. F. Punishment of saccharin drinking by amphetamine in rats and its reversal by chlordiazepoxide. Journal of Comparative and Physiological Psychology, 1973, 85, 97-104.

CASPER, N. J., \& REID, L. D. Complex contingencies. Physiological Psychology, 1975, 3, 9-13.

EDW ARDs, A. L. An introduction to linear regression and correlation. San Francisco: Freeman, 1976.

Farber, P. D., Gorman, J. E., \& REID, L. D. Morphine injections in the taste aversion paradigm. Physiological Psychology, 1976, 4. 365-368.

FARBER, P. D., \& REID, L. D. Addictive agents and intracranial stimulation (ICS): Daily morphine and pressing for combinations of positive and negative ICS. Physiological Psychology, 1976, 4, 262-268.

LeBlanc, A. E., \& CAPpell, H. Attenuation of punishing effects of morphine and amphetamine by chronic prior treatment. Journal of Comparative and Physiological Psychology, 1974, 87, 691-698.

Parker, L., Failor, A., \& Weidman, K. Conditioned preferences in the rat with an unnatural need state: Morphine withdrawal. Journal of Comparative and Physiological Psychology, 1973, 82, 294-300.

RILEY, A. L., \& BARIL, L. L. Conditioned taste aversion: A bibliography. Animal Learning \& Behavior, 1976, 4, 15-135. (Suppl)

Rossi, N. A., \& REID, L. D. Affective states associated with morphine injections. Physiological Psychology, 1976, 4, 269-274.

(Received for publication June 8, 1977; revision accepted October $14,1977$. 